The Astrophysical Journal, in Press

Preprint typeset using $\mathrm{LAT}_{\mathrm{E}} \mathrm{X}$ style emulateapj v. 5/2/11

\title{
A BROADBAND STUDY OF THE EMISSION FROM THE COMPOSITE SUPERNOVA REMNANT MSH 11-62
}

\author{
Patrick Slane, ${ }^{1}$ John P. Hughes, ${ }^{2}$ Tea Temim,${ }^{3}$ Romain Rousseau, ${ }^{4}$ Daniel Castro, ${ }^{1}$ Dillon Foight,${ }^{1}$ \\ B. M. Gaensler, ${ }^{5}$ Stefan Funk, ${ }^{6}$ Marianne Lemoine-Goumard, ${ }^{4}$ Joseph D. Gelfand ${ }^{7}$ David A. Moffett ${ }^{8}$ \\ Richard G. Dodson, ${ }^{9}$ And Joseph P. Bernstein ${ }^{10,11}$ \\ Accepted for Publication in ApJ
}

\begin{abstract}
MSH 11-62 (G291.1-0.9) is a composite supernova remnant for which radio and X-ray observations have identified the remnant shell as well as its central pulsar wind nebula. The observations suggest a relatively young system expanding into a low density region. Here we present a study of MSH 11-62 using observations with the Chandra, $X M M$, and Fermi observatories, along with radio observations from the Australia Telescope Compact Array (ATCA). We identify a compact X-ray source that appears to be the putative pulsar that powers the nebula, and show that the X-ray spectrum of the nebula bears the signature of synchrotron losses as particles diffuse into the outer nebula. Using data from the Fermi LAT, we identify $\gamma$-ray emission originating from MSH 11-62. With density constraints from the new X-ray measurements of the remnant, we model the evolution of the composite system in order to constrain the properties of the underlying pulsar and the origin of the $\gamma$-ray emission.
\end{abstract}

Subject headings: radiation mechanisms: non-thermal — ISM: supernova remnants: — ISM : individual (MSH 11-62) — stars: neutron

\section{INTRODUCTION}

The creation of an energetic pulsar in a supernova explosion often results in the formation of a composite supernova remnant (SNR) that can be characterized by emission from the remnant, an associated pulsar wind nebula (PWN), and the neutron star (NS) itself (see, e.g., Gaensler \& Slane 2006). Recent studies of such systems have resulted in discoveries of new pulsars, constraints on the spectra of particles injected from the pulsars into their nebulae, and evidence for interactions between the SNR reverse shock (RS) and the PWN. Broadband observations of composite SNRs have been crucial for improving our understanding of the detailed evolution of these systems, particularly with the vast improvements in capabilities provided by current X-ray and $\gamma$-ray observatories.

MSH 11-62 (Mills, Slee, \& Hill 1961) is a centrallybrightened SNR whose properties strongly suggest the

\footnotetext{
${ }^{1}$ Harvard-Smithsonian Center for Astrophysics, Cambridge, MA 02138-1516; slane@cfa.harvard.edu.

2 Dept. of Physics and Astronomy, Rutgers Univ., Piscataway, NJ 08854-8019; jph@physics.rutgers.edu.

3 NASA Goddard Space Flight Center - Code 662, Greenbelt, MD 20771; tea.temim@nasa.gov

${ }^{4}$ Université de Bordeaux, Centre d'Études Nucléaires Bordeaux Gradignan, CNRS-IN2P3, UMR 5797, Gradignan 33175, France; rousseau@cenbg.in2p3.fr, lemoine@cenbg.in2p3.fr

5 Sydney Institute for Astronomy, School of Physics A29, The University of Sydney, NSW 2006, Australia; bryan.gaensler@sydney.edu.au

${ }^{6}$ Kavli Institute for Particle Astrophysics and Cosmology, Stanford Linear Accelerator Center, Stanford, CA 94025, USA; funk@slac.stanford.edu

${ }^{7}$ New York University Abu Dhabi, P.O. Box 129188, Abu Dhabi, United Arab Emirates; jg168@astro.physics.nyu.edu

${ }^{8}$ Dept. of Physics, Furman Univ., Greenville, SC 29613; david.moffett@furman.edu.

${ }^{9}$ International Centre for Radio Astronomy Research University of Western Australia; richard.dodson@icrar.org.

${ }^{10}$ High Energy Physics Division, Argonne National Laboratory, Argonne, IL 60439; jpbernst@anl.gov.

${ }^{11}$ Department of Astronomy, University of Michigan, Ann Arbor, MI 48109
}

presence of a central nebula powered by an active pulsar. Radio observations reveal a remnant with a distinct bar-like central structure whose flat radio spectrum $\left(\alpha_{r}=0.29 \pm 0.05\right.$, where $\left.S_{\nu} \propto \nu^{-\alpha_{r}}\right)$ is similar to that observed for PWNe, and polarization measurements at 5 and $8.4 \mathrm{GHz}$ indicate that the magnetic field in the central region of the SNR is aligned with the long axis of the bar (Roger et al. 1986); the flux density at $8.4 \mathrm{GHz}$ is $10.4 \pm 0.4 \mathrm{Jy}$. No radio pulsar associated with MSH 1162 has been detected. An $11 \mathrm{hr}$ observation at $1.4 \mathrm{GHz}$ failed to detect any pulsations from this region, down to a sensitivity limit of $0.028 \mathrm{mJy}$ assuming a $10 \%$ duty cycle ${ }^{12}$.

The distance to MSH $11-62$ is poorly known. Limits on $\mathrm{HI}$ absorption indicate a distance greater than $3.5 \mathrm{kpc}$ (Moffett, Gaensler, \& Green 2001; Moffett et al. 2002). We adopt a fiducial distance of $5 \mathrm{kpc}$ in derived quantities below, and scale values to $d_{5}$, the distance in units of $5 \mathrm{kpc}$. Based on the radio synchrotron spectrum, the minimum-energy magnetic field in the central nebula has been estimated as $B \sim 65 d_{5}^{-2 / 7}\left(\theta_{P} / 2^{\prime} .5\right)^{-6 / 7} \mu \mathrm{G}$, where $\theta_{P}$ is the PWN radius in arcmin (Harrus, Hughes, \& Slane 1998).

X-ray emission from MSH 11-62 was first identified by Wilson (1986) using the Einstein Observatory. X-ray observations with the $A S C A$ observatory reveal a spectrum best described by a combination of thermal and nonthermal models (Harrus, Hughes, \& Slane 1998). The hard X-ray emission is spatially concentrated in the central regions, providing further evidence for the presence of a PWN within the remnant, but the limited angular resolution of $A S C A$ precluded detection of the pulsar itself. The derived spectral index for the X-ray power law component is $\Gamma=2.0_{-0.3}^{+0.1}$ and the associated luminosity is $L_{x}(0.2-4 \mathrm{keV}) \sim 6 \times 10^{33} d_{5}^{2} \mathrm{erg} \mathrm{s}^{-1}$. The best-fit temperature of the thermal component, presumably associated with emission from shock-heated gas in the SNR

\footnotetext{
${ }^{12}$ F. Camilo, private communication.
} 


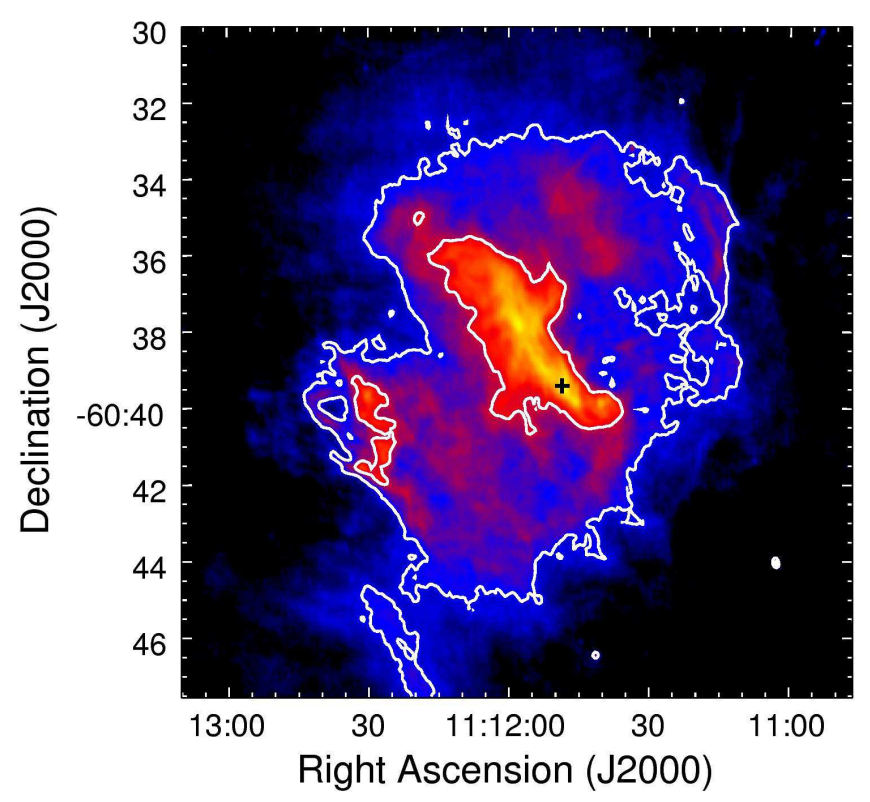

FIG. 1.- ATCA $1.4 \mathrm{GHz}$ image of MSH 11-62. The remnant is characterized by a faint shell with a bright central bar. Contours levels are 3.6 and $1.5 \mathrm{mJy}^{\text {beam }}{ }^{-1}$. The cross marks the position of the X-ray point source described in Section 3.

shell, is $k T \sim 0.8 \mathrm{keV}$. These observations clearly imply that MSH 11-62 harbors a young pulsar that powers a central PWN. Chandra observations, which we discuss in detail below, reveal a compact X-ray source that is almost certainly the pulsar counterpart (Harrus et al. 2002; Kargaltsev \& Pavlov 2008).

Observations with the Compton Gamma-Ray Observatory discovered the source 3EG J1102-6103, for which MSH 11-62 falls within the position error circle. The flux is $F(>100 \mathrm{MeV})=(3.3 \pm 0.6) \times 10^{-7}$ photons $\mathrm{cm}^{-2} \mathrm{~s}^{-1}$, with a power law index $\Gamma=2.5 \pm 0.2$ (Hartman et al. 1999). This source was suggested as a $\gamma$-ray counterpart to MSH 11-62 (Sturner \& Dermer 1995), but was also subsequently suggested as the counterpart to the nearby young pulsar J1105-6107 (Kaspi et al. 1997). Given the large uncertainty in the position of the $\gamma$-ray source, the origin of its emission remained uncertain.

Here we report on observations with the ATCA, the Chandra X-ray Observatory, the XMM-Newton X-ray Observatory, and the Fermi Gamma-Ray Space Telescope that provide sufficient sensitivity and resolution to identify the neutron star in MSH 11-62, and to provide a much-improved characterization of the properties of this composite system. In Sections 2 we discuss our ATCA observations. In Section 3 we summarize the Chandra and $X M M$ observations, with detailed investigations of the PWN, neutron star, and SNR shell. In Section 4 we discuss observations with the Fermi LAT, and in Section 5 we present discussions of the results along with our conclusions.

\section{RADIO OBSERVATIONS}

\subsection{Observations and Data Reduction}

Radio observations of MSH 11-62 were carried out with the ATCA, a 6-element interferometer located near Narrabri, New South Wales, Australia. Observations were carried out on three separate dates using three different array configurations: on 1999 Feb 10 (750C array),

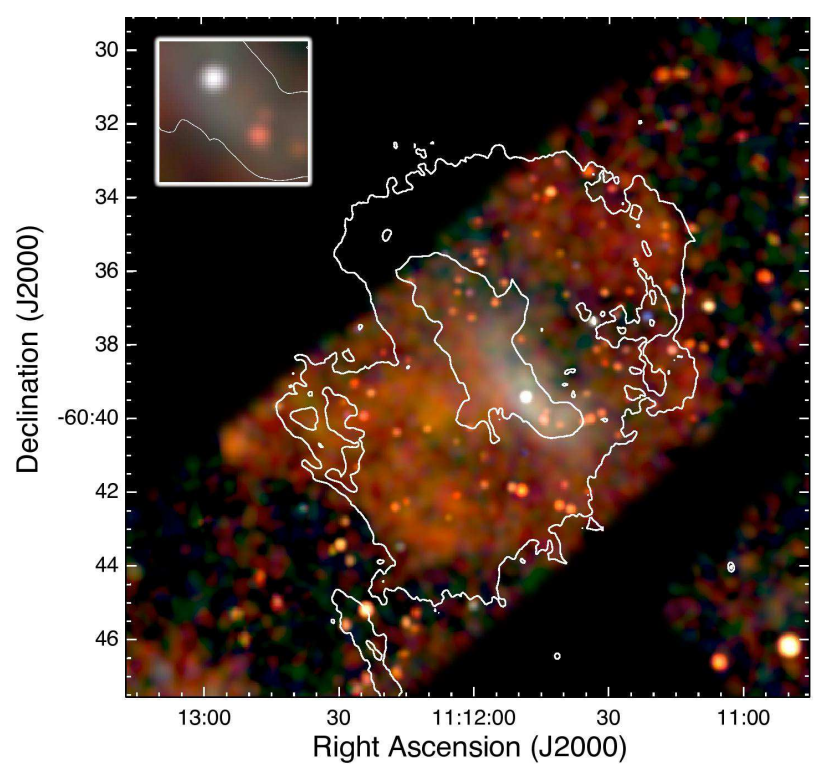

Fig. 2.- Chandra image of MSH 11-62. Colors correspond to different X-ray energy bands: red $=0.75-1.45 \mathrm{keV}$; green $=1.45$ $-2.58 \mathrm{keV}$; blue $=2.58-6.0 \mathrm{keV}$. The SNR shell is evident as soft (red) X-ray emission (the portion of the shell in the northeast falls outside the detector field of view). The central bar and associated compact source are white in color, corresponding to harder emission. The inset figure shows the central regions of the PWN containing the pulsar candidate (white) and the star Tycho 8959 2551 (red).

1999 Feb 22 (6C array), and 1999 Mar 24 (1.5B array). In all cases, radio continuum data were recorded in all four polarizations, over a bandwidth of $128 \mathrm{MHz}$ centered at $1384 \mathrm{MHz}$. At each epoch, MSH 11-62 was observed for 8-11 hours spread over a 12-hour synthesis, combined with regular short observations of the extragalactic source PKS B1036-697 to calibrate atmospheric gain variations and polarization leakages. The bright source PKS B1934-638 was also observed at each epoch to provide an overall flux calibration.

Following editing of the data and calibration in the MIRIAD package using standard techniques, the visibility data-set was imaged using multi-frequency synthesis and uniform weighting, and then deconvolved with a maximum entropy algorithm. The final total intensity image has an angular resolution of $8 . .8 \times 6.6$, with the major axis oriented at a position angle $3^{\circ}$ east of north, and an RMS sensitivity of $0.1-0.2 \mathrm{mJy}$ beam $^{-1}$. Preliminary investigations of these observations have been presented by Moffett, Gaensler, \& Green (2001) and Moffett et al. (2002).

\subsection{Radio Structure and Properties}

The ATCA $1.4 \mathrm{GHz}$ image of MSH 11-62 (Figure 1) reveals a roughly circular morphology with a bright central bar-like structure that corresponds with the flatspectrum region identified by Roger et al. (1986). Partial limb-brightening is observed along the northwestern and southeastern boundaries of the SNR shell. Considerable filamentary structures are observed both along the limb and in the SNR interior. The bright central bar reveals several regions of enhanced radio emission, with the brightest feature located near the southwestern end of the structure (at an approximate position of $\left.11^{\mathrm{h}} 11^{\mathrm{m}} 48^{\mathrm{s}},-60^{\circ} 39^{\prime} 27^{\prime \prime}\right)$. The structure of the central bar 
is similar to that observed in several other PWNe, including G11.2-0.3 (Tam et al. 2002), N157B (Lazendic et al. 2000), and G328.4+0.2 (Johnston, McClureGriffiths, \& Koribalski 2004; Gelfand et al. 2007). Its apparent similarity to the PWN in G328.4+0.2, in particular, may suggest that the nebula has been compressed by the SNR reverse shock (RS), which may have propagated more quickly from the NW/SE directions, where the SNR limbs show slight brightness enhancements.

The total $1.4 \mathrm{GHz}$ flux from the SNR is $14 \mathrm{Jy}$, with $4.8 \mathrm{Jy}$ associated with the central nebula. This revised flux value results in a new minimum-energy estimate for the magnetic field: $B \approx 36 d_{5}^{-2 / 7}\left(\theta_{P} / 2^{\prime} .5\right)^{-6 / 7} \mu \mathrm{G}$. As we discuss in Section 5.3, however, some evolved PWNe are known to be particle-dominated, with magnetic field strengths far below the minimum-energy (or equipartition) values.

\section{X-RAY OBSERVATIONS}

A $50 \mathrm{ks}$ Chandra ACIS observation of MSH 11-62 was performed on 2002 April 8 (ObsID 2782). The central region of the SNR was placed on the S3 chip of the detector, and portions of the shell were imaged on the adjacent chip S2. The extent of the SNR (with a radius $R_{\mathrm{SNR}} \approx 5.7$ arcmin) is considerably larger than the field covered by the S3 chip, which resulted in the northeastern regions of the remnant falling outside of the detector field of view.

MSH 11-62 was observed for $40 \mathrm{ks}$ with $X M M$, on 2002 February 6 (ObsID 0051550101). The MOS2 and pn detectors were operated in full frame mode with the MEDIUM filters, providing full coverage of the SNR, while MOS1 was operated in timing mode with the THIN filter.

\subsection{Observations and Data Reduction}

The Chandra data were reduced using standard screening prescriptions. All data were reduced using tools from $C I A O$ 3.2.2.. After screening, the remaining exposure time was $49.4 \mathrm{ks}$.

The Chandra observations show hard emission extended along the radio bar (Figure 2), although the emission does not extend as far to the $\mathrm{NE}$ as the radio bar. There is also a clear shell of soft X-ray emission that corresponds well with the morphology of the radio SNR, including a small intensity enhancement along the position of the western radio limb-brightening, and a large underluminous region to the northwest of the radio bar.

Of particular significance in the Chandra image is the presence of numerous point sources of X-ray emission along the line of sight to MSH 11-62. These appear to be associated with the open cluster $\operatorname{Tr} 18$, located at a distance of $\sim 1.5 \mathrm{kpc}$ (Vazquez \& Feinstein 1990).

We have analyzed spectra from both the central X-ray bar and the SNR shell using background regions selected from regions adjacent to the bar, and just outside boundaries of the remnant, respectively. Weighted response matrix (RMF) and ancillary response (ARF) files were created for the extended regions, and spectral modeling was then carried out using XSPEC version 11.3.2ag. Point sources within in the regions of interest were excluded from the spectral extraction.

The $X M M$ data were reduced using SAS Version 10.0.

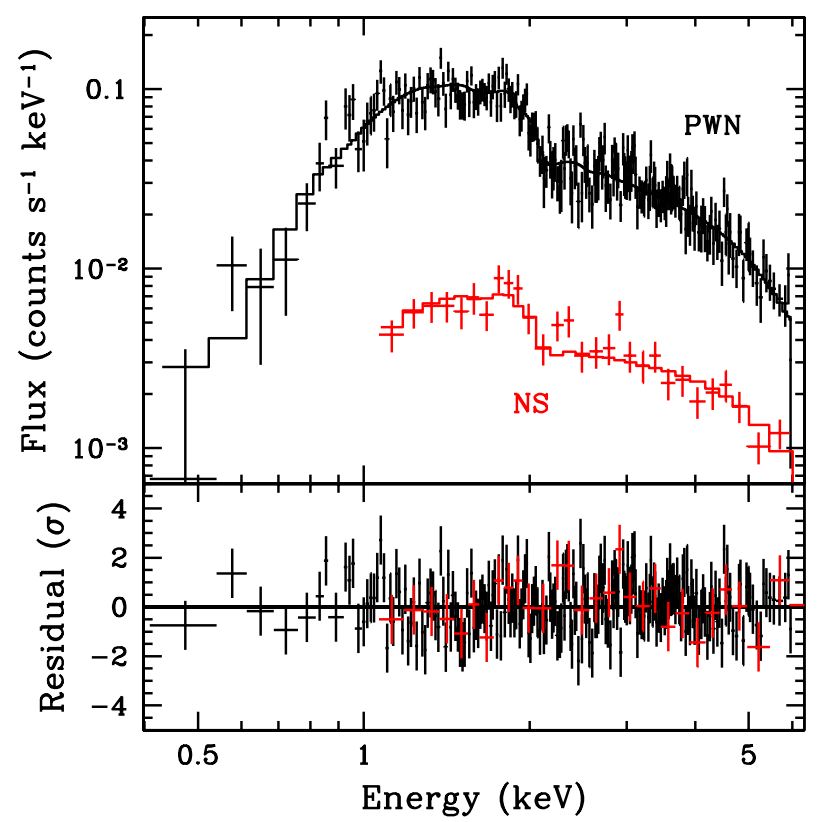

FIG. 3.- Chandra ACIS X-ray spectra for PWN (black) and NS (red) with absorbed power law fits.

Standard screening resulted in cleaned exposures of 35.6 (29.2) ks for MOS2 (pn).

\subsection{Central Bar}

The central X-ray bar is aligned with the radio bar, and extends to the edge of the radio in the southwest, but the brightness falls off more quickly than the radio emission in the northeast direction. There is a bright X-ray source located at the position of the brightest radio emission, likely representing the pulsar that powers the nebula. The source, located at $11^{\mathrm{h}} 11^{\mathrm{m}} 48^{\mathrm{s}} \cdot 62,-60^{\circ} 39^{\prime} 26^{\prime \prime} .2$ (and hereafter identified as CXOU J111148.6-603926), is roughly centered on the X-ray nebula. A fainter point source is detected at the southwestern edge of the radio bar, with coordinates $11^{\mathrm{h}} 11^{\mathrm{m}} 44^{\mathrm{s}} .5,-60^{\circ} 40^{\prime} 02^{\prime \prime} .8$ (see inset to Figure 2). The source is positionally coincident with the $V=10.8$ star Tycho 89592551 , which is unrelated to the nebula.

The Chandra spectrum of the X-ray bar (excluding CXOU J111148.6-603926) is well-described by a power law model with $N_{H} \approx 7 \times 10^{21} \mathrm{~cm}^{-2}$ and $\Gamma \approx 1.7$ (Table 1, Figure 3), securing the PWN interpretation for this structure. Here we have used an elliptical background region on S3, adjacent to the PWN but within the SNR, to account for both sky and instrumental background as well as thermal emission from the SNR. An independent fit using the $X M M$ pn data yields virtually identical results. The luminosity $(0.5-10 \mathrm{keV})$ is $1.1 \times 10^{34} d_{5}^{2} \mathrm{erg} \mathrm{s}^{-1}$, about a factor of two higher than the value determined from $A S C A$ observations (Harrus, Hughes, \& Slane 1998), but far lower than the value of $\sim 8 \times 10^{34} d_{5}^{2} \mathrm{erg} \mathrm{s}^{-1}$ cited by Kargaltsev \& Pavlov (2008).

To search for spectral variations in the PWN, we extracted spectra from rectangular regions along the nebula, as shown in Figure 4. We fixed the column density to that obtained for the entire PWN, and then fit each spectrum to a power law model. The results, summarized in 
TABLE 1

Spectral Fit Parameters

\begin{tabular}{cccc}
\hline \hline Region & Data & Parameter & Value \\
\hline PWN & CXO & $N_{H}$ & $(6.7 \pm 0.7) \times 10^{21} \mathrm{~cm}^{-2}$ \\
(whole) & & $\Gamma$ & $1.8 \pm 0.1$ \\
& & $F_{x}^{a}$ & $3.5 \times 10^{-12} \mathrm{erg} \mathrm{cm}^{-2} \mathrm{~s}^{-1}$ \\
PWN & CXO & $N_{H}^{a}$ & $6.7 \times 10^{21} \mathrm{~cm}^{-2}$ \\
Region 1 & & $\Gamma$ & $1.7 \pm 0.1$ \\
Region 2 & & & $1.8 \pm 0.1$ \\
Region 3 & & & $2.2 \pm 0.1$ \\
Region 4 & & & $1.5 \pm 0.1$ \\
Region 5 & & & $2.5 \pm 0.1$ \\
Region 6 & & & $6.7 \times 10^{21} \mathrm{~cm}^{-2}$ \\
& & $N_{H}^{a}$ & $1.2 \pm 0.2$ \\
NS & $\mathrm{CXO}$ & $\Gamma$ & $3.7 \times 10^{-13} \mathrm{erg} \mathrm{cm}^{-2} \mathrm{~s}^{-1}$ \\
& & $F_{x}^{b}$ & $6.7 \times 10^{21} \mathrm{~cm}^{-2}$ \\
& & $N_{H}^{a}$ & $1.3_{-0.2}^{+0.5} \mathrm{keV}^{10}$ \\
SNR & $\mathrm{XMM}$ & $k T_{1}$ & $(1.5 \pm 0.2) \times 10^{10} \mathrm{sm}^{-3}$ \\
(whole) & & $n_{e} t$ & $2.8 \pm 0.4 \mathrm{keV}^{-2} \mathrm{~s}^{-1}$ \\
& & $k T_{2}$ & $2.1 \times 10^{-11} \mathrm{erg} \mathrm{cm}^{-2} \mathrm{~s}^{b}$ \\
& & $F_{1}^{b}$ & $3.0 \times 10^{-12} \mathrm{erg} \mathrm{cm}^{-2} \mathrm{~s}^{-1}$
\end{tabular}

Note. -

a) Fixed at best-fit value for PWN

b) Unabsorbed $0.5-10.0 \mathrm{keV}$ flux.

Table 1, show a spectral steepening with distance from the central neutron star. Such steepening is also observed in G21.5-0.9 (Slane et al. 2000), 3C 58 (Bocchino et al. 2001; Slane et al. 2004) and other PWNe, and is understood to be associated with synchrotron aging of the particles as they diffuse from the injection point to the outer nebula (Kennel \& Coroniti 1984), although models matching the exact radial behavior of the spectral index remain elusive (e.g. Reynolds 2003).

\subsection{Compact Source}

Our Chandra observations obtain 820 backgroundsubtracted counts from within a circle with a radius of 3.3 arcsec centered on the compact source CXOU J111148.6603926. The associated count rate of $1.6 \times 10^{-2} \mathrm{cnt} \mathrm{s}^{-1}$ yields negligible pileup in the image. The radial profile of the compact source image is well-described by the point spread function of the telescope, with no evidence of a surrounding jet/torus structure as is seen in many other such systems, although evidence for filamentary structure is seen on larger scales (see Figure 4).

The spectrum of the compact source is well-described by a power law with the column density fixed at the value obtained from fits of the entire PWN, and with $\Gamma=$ $1.2 \pm 0.2$. The unabsorbed flux is $F_{x}(0.5-10 \mathrm{keV})=3.7 \times$ $10^{-13} \mathrm{erg} \mathrm{cm}^{-2} \mathrm{~s}^{-1}$ indicating a luminosity $L_{x}(0.5-$ $10 \mathrm{keV})=1.1 \times 10^{33} d_{5}^{2}$ erg $\mathrm{s}^{-1}$, similar to that measured for a number of other young pulsars with bright PWNe (see Kargaltsev \& Pavlov 2008).

\subsection{SNR Shell}

To investigate the X-ray properties of the SNR shell, we extracted spectra of the entire SNR from both the

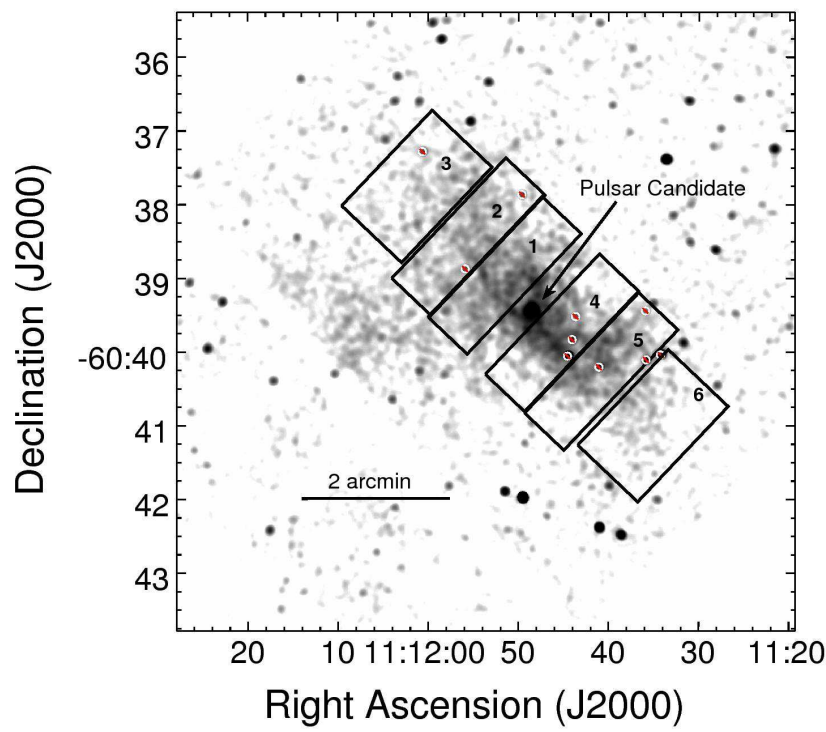

Fig. 4.- Chandra image of PWN in MSH 11-62, along with regions used for extracting the spectral index along the nebula (see Table 1). The image has been smoothed with a Gaussian of 5 arcsec width. The pulsar candidate is the bright central source between regions 1 and 4 . White circles with red slashes indicate point sources removed before extraction of spectra.

$X M M$ pn and MOS2 detectors (see Figure 5). Emission from the PWN was eliminated from the spectra, as was emission from point sources in the field. Weighted response files were generated for each spectrum, and background spectra were obtained from regions outside the SNR shell in each detector.

The spectra (Figure 6) were fit simultaneously to an absorbed nonequilibrium ionization planar shock model (VPSHOCK in XSPEC), with the column density fixed at the best-fit value for the PWN. We find an electron temperature $k T=1.5_{-0.2}^{+0.5} \mathrm{keV}$ and an ionization timescale $n_{e} t=(2.9 \pm 0.9) \times 10^{10} \mathrm{~s} \mathrm{~cm}^{-3}$, with evidence for mild overabundances of $\mathrm{Ne}, \mathrm{Mg}$, and $\mathrm{Si}$. The fit requires a second, harder component, which can be fit with a power law with $\Gamma=2.6 \pm 0.2\left(\chi_{r}^{2}=1.3\right.$ with 612 degrees of freedom), but a slightly better fit $\left(\Delta \chi^{2}=6.1\right.$ for the same number of degrees of freedom) is obtained with a second thermal component (NEI model in XSPEC) with $k T=2.8 \pm 0.4 \mathrm{keV}$ and cosmic abundances. If associated with the forward shock, this electron temperature corresponds to an expansion speed of $\sim 1600 \mathrm{~km} \mathrm{~s}^{-1}$ assuming temperature equilibration between electrons and ions (and a higher speed if equilibration has not yet been reached).

Based on the volume emission measure for the lowertemperature component, and assuming a thin-shell morphology for the SNR, with a shell thickness of $R / 12$ corresponding to a shock compression ratio of 4 , the estimated preshock density is $n_{H}=0.04 \eta^{1 / 2} d_{5}^{-1 / 2} f^{-1 / 2} \mathrm{~cm}^{-3}$, where $\eta$ is the fraction of the X-ray emission that is not associated with point sources in the field, and $f$ is the fraction of the remnant shell actually seen in X-rays. The observed variation in brightness around the SNR shell suggests possible density variations of a factor of two or so, and we consider the range $0.02-0.08 \mathrm{~cm}^{-3}$ in modeling the dynamical evolution in Section 5. We note that the higher temperature component provides very nearly 


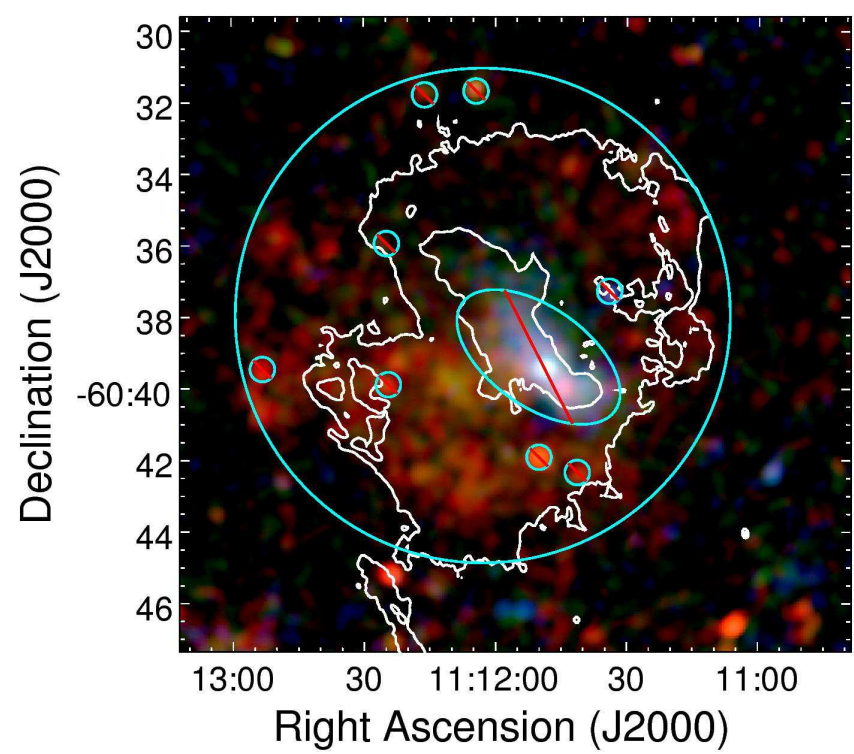

Fig. 5.- XMM MOS2 image of MSH 11-62, with radio contours from Figure 1. The image has been exposure-corrected, and $\mathrm{red} /$ green/blue corresponds to the energy band 0.6-1.5/1.5-2.5/2.5$5.0 \mathrm{keV}$. The large circle indicates the region from which the SNR spectrum was extracted. Events from the inner ellipse were ignored to eliminate the contribution from the PWN, and those from the small circles with red slashes were ignored to reduce point source contributions. The same inner ellipse was used to extract the spectrum of the PWN.

the same density estimate as that from the low temperature component.

The mass swept up by the SNR under such a scenario is then $M_{s w}=0.5 \eta^{1 / 2} f^{1 / 2} d_{5}^{5 / 2} M_{\odot}$. Given that the system morphology appears to show evidence that the PWN has undergone an interaction with the SNR reverse shock, this low value for the swept-up mass would appear to suggest a rather low ejecta mass for the supernova. The inferred density, combined with the ionization timescale from the spectral fits, indicates an age of $5800 \eta^{-1 / 2} d_{5}^{1 / 2} f^{1 / 2} \mathrm{yr}$, which should be considered an upper limit since portions of the SNR shell that are not detected in X-rays presumably have lower density values.

\section{GAMMA-RAY OBSERVATIONS}

\subsection{Fermi LAT}

We investigated $\gamma$-ray events acquired from the region surrounding MSH 11-62 with the Fermi-LAT during the period 2008 August 5 to 2011 March 14. Standard event selection was applied, using "Source" class events with zenith angles less than 100 degrees to minimize the portion of the Earth limb in the LAT field of view (Abdo et al. 2009). The Pass 7 version 6 instrument response functions were used, along with standard analysis tools available from the Fermi Science Support Center (version v9r21p0).

Standard background models were used to account for both Galactic and extragalactic diffuse emission as well as instrumental background. Sources within $10^{\circ}$ of MSH 11-62, with a statistical significance larger than $5 \sigma$ above the background, are extracted from the twoyear Fermi-LAT Second Source Catalog (Abdo et al. 2011), except for 2FGL J1112.1-6040 which corresponds

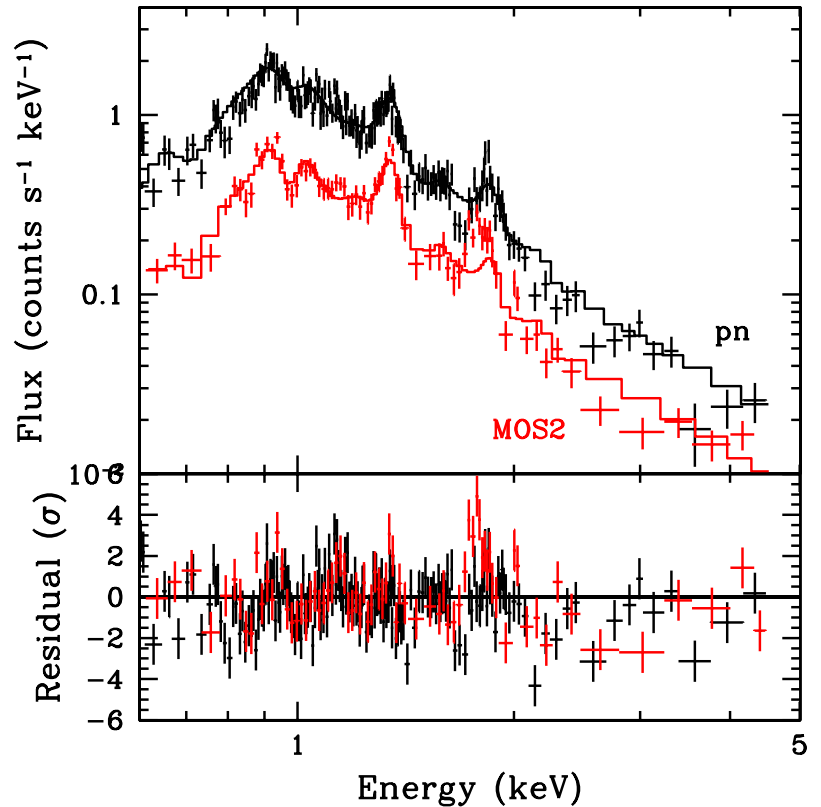

FIG. 6.- XMM spectra from the SNR shell of MSH 11-62, with pn data shown in black and MOS2 data shown in red. The best-fit VPSHOCK model is shown as a histogram.

to the $\gamma$-ray emission of MSH 11-62. The flux parameters for all the point-like sources less than $5^{\circ}$ from MSH 11-62 are left free in the likelihood fit while the spectral parameters of other sources are fixed at the 2FGL values. The mapcube file ring_2year_P 76_v0.fits was used to describe the Galactic $\gamma$-ray emission, and the isotropic component was modeled using the isotrop_2year_Pr6_source_v0.txt table. Data analysis details follow those in Castro \& Slane (2010) and Grondin et al. (2011).

Using the binned maximum likelihood analysis package gtlike, the Test Statistic, TS, (for which the significance is approximately $(T S)^{1 / 2} \sigma$; Mattox et al 1996, Equation 20) for MSH 11-62 was found to be 432 when the source was analyzed in the energy range from $100 \mathrm{MeV}$ to 100 $\mathrm{GeV}$. This value of the Test Statistic corresponds to a 21 sigma detection of the source. A map of the test statistic as a function of position, used to localize the best fit position of MSH 11-62, is shown in Figure 7. Overlaid on this plot are blue contours corresponding to ATCA observation of MSH 11-62 and white contours corresponding to the LAT significance levels of $\sim 13,15$, and $17 \sigma$.

We also indicate the positions of the three known pulsars within a $45^{\prime}$ radius from the centroid of the LAT source. If each of these pulsars converts its entire spindown power into $\gamma$-rays in the LAT band, they could account for no more than $6 \%$ of the observed flux, and we thus ignore their contributions in the subsequent analysis. The nearest pulsar with a high $\dot{E} / d^{2}$ ratio is PSR J1105-6107, located 55' from the centroid of the $\gamma$-ray emission, well beyond the border of Figure 7 ; we consider an association with this source to be very unlikely.

The source spectrum was extracted using both front and back events, over the energy range 0.1-100 GeV. The spectrum is shown in Figure 8, and is well-described 


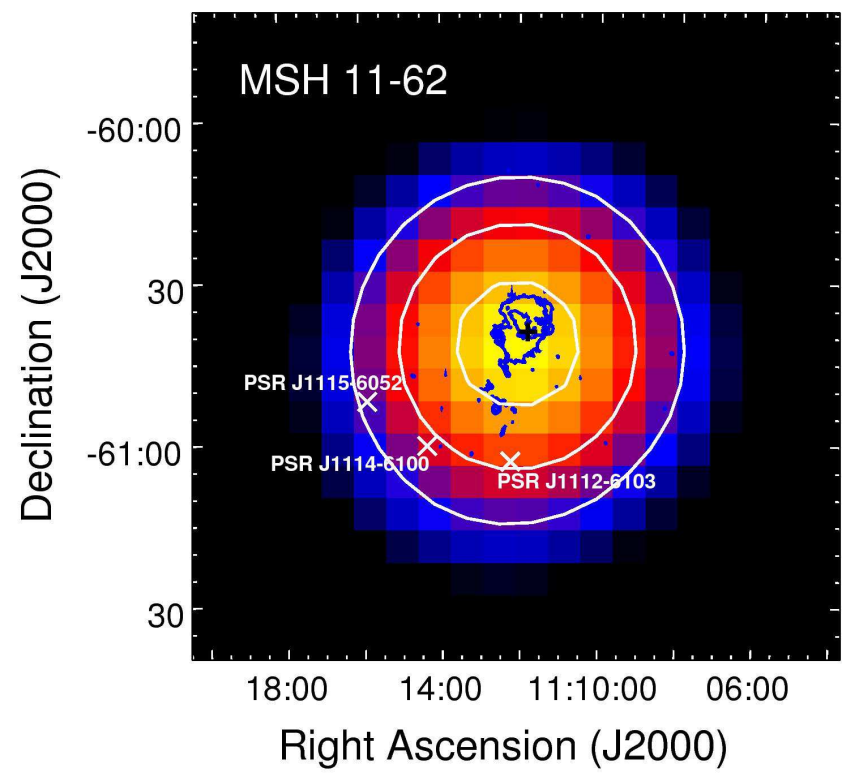

FIG. 7.- TS map of Fermi LAT emission from MSH 11-62. Blue contours correspond to the radio emission, and white contours represent the 13,15 , and $17 \sigma$ significance levels of the flux. Positions of nearby pulsars with $\dot{E}>10^{32} \mathrm{erg} \mathrm{s}^{-1}$ are indicated (see discussion in text).

by a power law with $\Gamma=1.2 \pm 0.2$ accompanied by an exponential cutoff with $E_{\text {cut }}=3.2 \pm 0.9 \mathrm{GeV}$ that improves the fit by $\sim 6.8 \sigma$. The associated flux is $F(\geq$ $100 \mathrm{MeV})=(6.3 \pm 1.2 \pm 3.2) \times 10^{-8}$ photons $\mathrm{cm}^{-2} \mathrm{~s}^{-1}$ [or $(8.0 \pm 0.6 \pm 4.2) \times 10^{-11} \mathrm{erg} \mathrm{cm}^{-2} \mathrm{~s}^{-1}$, where the quoted uncertainties are statistical and systematic, respectively.

Two main systematic uncertainties can affect the LAT flux estimation for a point source in the Galactic plane: uncertainties on the Galactic diffuse background and on the effective area. The dominant uncertainty at low energy comes from the Galactic diffuse emission, which we estimated by changing the normalization of the Galactic diffuse model artificially by $6 \%$ as done in Abdo et al. (2010). The second systematic is estimated by using modified IRFs whose effective areas bracket those of our nominal Instrument Response Function (IRF). These bracketing IRFs are defined by envelopes above and below the nominal energy dependence of the effective area by linearly connecting differences of $(10 \%, 5 \%, 20 \%)$ at $\log (\mathrm{E})$ of $(2,2.75,4)$, respectively.

The source 2FGL J1112.1-6040, from the LAT $2^{\text {nd }}$ year catalog, was identified with a possible association with MSH 11-62(Abdo et al. 2011). The 2FGL flux agrees with our measurements as well, but both are significantly lower than that of 3EG J1102-6103, whose quoted spectral index is also significantly steeper than that for MSH 11-62. These differences are presumably associated with the previously-unrecognized spectral cutoff.

We have searched for possible extension of the source using the routine POINTLIKE (Kerr 2011), comparing the test statistic $T S_{\text {ext }}$ for the extended hypothesis to that for a point source hypothesis, $T S_{p}$, defining $T S_{\text {ext }}=$ $T S-T S_{p}$. We selected events converting in the front section of the tracker, where the Point Spread Function (PSF) is the narrowest. We fitted the position of the source assuming iteratively a point source and a Gaussian

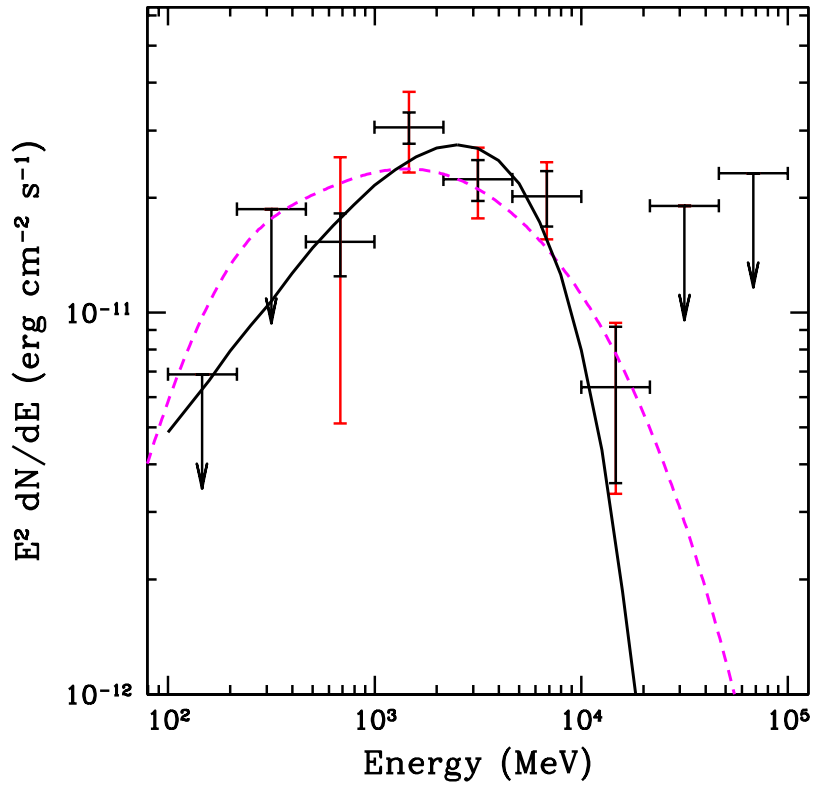

FIG. 8.- Fermi LAT spectrum of MSH 11-62. Black error bars correspond to statistical errors, while red error bars include systematic errors as well. The solid curve corresponds to the best-fit model for a power law with an exponential cutoff. The dashed magenta curve corresponds to a pion-decay model assuming a power law proton spectrum with an exponential cutoff. See Section 5.2 for discussion.

shape for which we fitted the extension. We find that the best-fit centroid is located at $11 \mathrm{~h} 12 \mathrm{~m} 12 \mathrm{~s},-60^{\circ} 40^{\prime} 12^{\prime \prime}$, in good agreement with the position of MSH 11-62, and that there is no evidence for extent with significance greater than $3 \sigma$. Computing a significance map, the POINTLIKE routine determines the likelihood gradient with spatial coordinates; this gradient has a $1 \sigma$ width of 1.2 arcmin in each direction for MSH 11-62. The significance contours thus provide an indirect way of characterizing the statistical uncertainties on the position of the detected source. A deeper observation is required to firmly address, with higher confidence, whether or not the Fermi LAT source is extended.

\subsection{H.E.S.S. Upper Limits}

The position of MSH 11-62 was covered in a survey of the inner Galactic plane with the High Energy Spectroscopic System (H.E.S.S.) in 2004/2005. The approximate exposure time on the source was $9.1 \mathrm{hr}$ (Hoppe 2008), resulting in no reported detection of the source. Based on this non-detection, we calculate a $2 \sigma$ upper limit on the VHE flux at $1 \mathrm{TeV}$ for MSH 11-62 by scaling the H.E.S.S. sensitivity for a $5 \sigma$ point source detection $(1 \%$ of the Crab in $25 \mathrm{~h}$ under the assumption of a photon index of 2.6) to the actual H.E.S.S. exposure for the source. We find $E^{2}(d N / d E)_{E=1 T e V}<3.8 \times 10^{-12} \mathrm{erg} \mathrm{cm}^{-2} \mathrm{~s}^{-1}$, where $N$ is the number of photons per unit area per second.

\section{ANALYSIS AND DISCUSSION \\ 5.1. Evolutionary State}

The large-scale evolution of a composite SNR depends on the mechanical energy of the explosion, the density of the ambient medium, the mass of the supernova ejecta, 


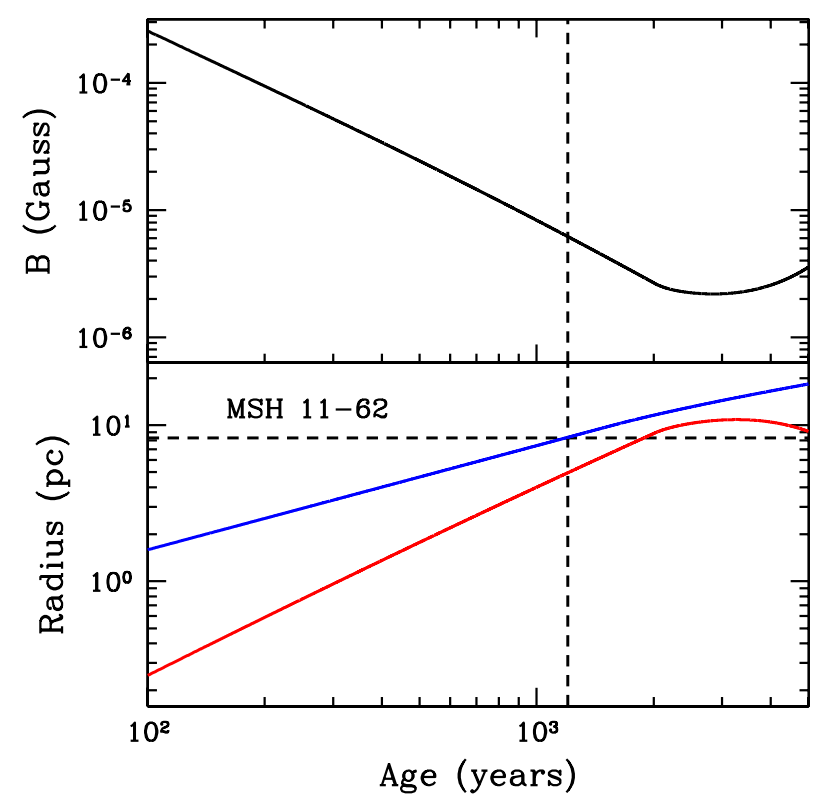

FIG. 9.- Upper: Time evolution of the PWN magnetic field. Lower: Evolution of the SNR (blue) and PWN (red) radii as a function of time. The observed radius of MSH 11-62 is indicated, and the vertical line indicates the age at which this radius is reached. See Section 5.1 for model details.

and the spin-down power of the pulsar. Additional parameters of considerable importance are the braking index and spin-down timescale of the pulsar, along with the fraction of the spin-down power that appears as magnetic flux.

Figure 9 shows an illustrative model for the evolution of MSH 11-62 using parameters for a scenario described in Section 5.3. For these parameters, the forward shock (FS) of the SNR (blue curve) reaches the observed radius of MSH $11-62\left(R_{S N R} \approx 8.3 d_{5} \mathrm{pc}\right)$ at an age of $\sim 1.2 \mathrm{kyr}$. By this time, the reverse shock has begun to approach the PWN (for which the model yields $R_{P W N} \approx 5 \mathrm{pc}$, or an angular diameter of $\approx 6.9$ arcmin at a distance of $5 \mathrm{kpc}$ ), as indicated by the subsequent compression of the PWN radius (red curve). This is consistent with the somewhat distorted morphology of MSH 11-62, which appears to indicate that the RS has propagated most rapidly along the NW/SE direction. In addition, Figure 9 shows that the magnetic field in the PWN has declined to a value of $\sim 6 \mu \mathrm{G}$.

The broadband emission observed from MSH 11-62 places considerable constraints on the underlying properties of the composite system. Of particular importance is our understanding of the observed $\gamma$-ray emission, which could originate from the pulsar, the PWN, or the SNR itself. Here we consider models for each scenario using constraints derived from the radio and X-ray observations of MSH 11-62. In these model investigations, we have varied input parameters to investigate the broad effects they have on the broadband spectrum, and have then fine-tuned parameters to provide reasonable agreement with the data. The resulting models are not statistical fits to the observed emission, and were not optimized by a likelihood analysis over the large parameter space.

\subsection{Gamma-rays from the SNR}

Particle acceleration at the forward shocks of SNRs is known to produce extremely energetic particles that can produce $\gamma$-rays through inverse-Compton (IC) scattering of ambient photons by energetic electrons, and/or through the decay of neutral pions produced in energetic proton-proton collisions. Thus, one interpretation for the $\gamma$-ray emission from MSH $11-62$ is that it originates from the SNR itself, and is a signature of particle acceleration by the remnant. We consider two cases, one in which the $\gamma$-ray emission is dominated by hadrons $\left(\pi^{0}\right.$-decay), and one in which it is dominated by leptons (IC scattering).

For the hadronic-dominated scenario, we assume that the mechanical energy of the SNR is $E_{S N R}=10^{51} \mathrm{erg}$ and that $50 \%$ of this energy is converted to energetic particles whose spectrum is described by a power law with an exponential cutoff. We assume an electron-to-proton ratio $k_{e-p}=10^{-2}$, and that the spectral index is the same for protons and electrons. Using $d=5 \mathrm{kpc}$, we adjust the spectral index, density, and cutoff energy for the proton spectrum to match the Fermi LAT spectrum, while also adjusting the magnetic field strength to reproduce the SNR radio emission with synchrotron emission from the electron spectrum. We find a spectral index of 1.8 and an exponential cutoff energy of $70 \mathrm{GeV}$ for the protons (Figure 10, top), with an ambient density $n_{0}=6.8 \mathrm{~cm}^{-3}$. The associated $\gamma$-ray emission from IC scattering of the cosmic microwave background (CMB) is negligible. The postshock magnetic field required by the radio emission is $\sim 10 \mu \mathrm{G}$. Here we have used the measured 1.4 GHz flux from the shell, and assumed a spectral index range of $\alpha_{r}=0.4-0.7$ typical of radio emission from shell-type SNRs.

The required proton cutoff energy under this scenario is rather low given that we expect energies well in excess of $10^{12} \mathrm{eV}$ to be produced in young SNRs, but it is possible that a low shock speed has resulted in a low maximum energy (e.g., Reynolds 2008). Of potentially greater concern is that the preshock density required under this scenario is more than two orders of magnitude larger than that inferred from the X-ray spectrum. Similar discrepancies have been identified for several $\gamma$-ray emitting SNRs associated with $\mathrm{OH}$ masers (which imply an interaction between the SNRs and surrounding molecular clouds, thus making $\pi^{0}$-decay a likely production mechanism), and it has been suggested that this could be the result of a clumpy SNR shell in which only the lower-density interclump medium is responsible for the X-ray emission while all of the gas participates in the $\gamma$-ray production (Castro \& Slane 2010). No maser emission has been reported for MSH 11-62, and the remnant is not known to be interacting with dense material. Thus, while this scenario cannot be ruled out, particularly since the production of masers requires a rather narrow range of physical conditions, it does not have strong supporting evidence outside of the $\gamma$-ray emission. Searches for evidence of interactions between MSH 11-62 and molecular clouds are of particular importance to further address this picture.

For at least some SNRs, the observed $\gamma$-ray emission appears to be dominated by IC emission from an energetic population of electrons (e.g., Ellison et al. 2010). We have considered such a scenario for MSH 11-62 by fixing the ambient density at the value implied by the $\mathrm{X}$ - 


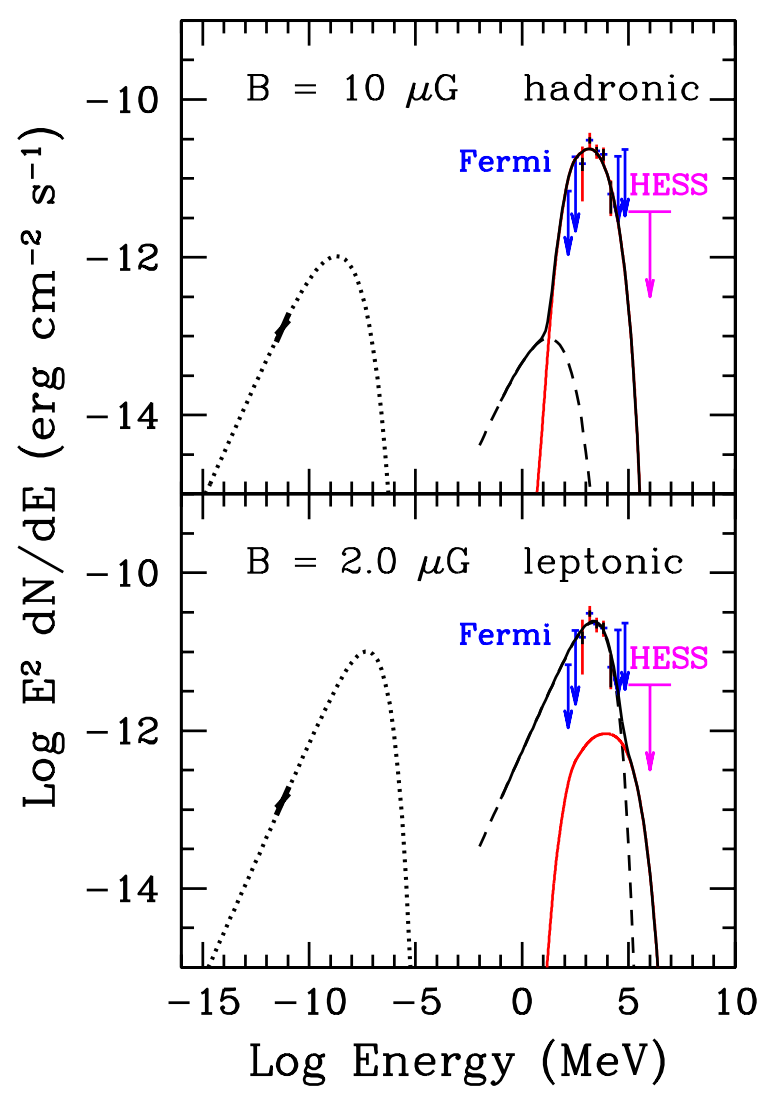

FIG. 10.- Models for the broadband emission from MSH 1162 under the assumption of dominant hadronic (upper) and leptonic (lower) processes for the observed $\gamma$-ray emission. The dotted curves correspond to synchrotron emission, and the dashed curves correspond to IC emission. The red curve represents $\gamma$-ray emission from pion decay, and the solid black line represents the summed $\gamma$-ray components.

ray measurements, and allowing the normalization and shape of the electron spectrum to vary. We find that for a distance of $5 \mathrm{kpc}$, the total electron energy required to produce the $\gamma$-ray emission through IC scattering of $\mathrm{CMB}$ photons is $8 \times 10^{49} \mathrm{erg}$. The magnetic field required to fit the radio emission from the SNR shell is $B=2.0 \mu \mathrm{G}$. The model, shown in Figure 10 (bottom), requires a super-exponential cutoff of the electron spectrum

$$
N_{e} \propto E_{e}^{-\alpha_{e}} \exp \left[-\left(\frac{E_{e}}{E_{c u t, e}}\right)^{b}\right]
$$

Model parameters are given in Table 2. In particular, we find $E_{c u t, e}=0.9 \mathrm{TeV}$, which is quite low given the rather low magnetic field. Since all realistic acceleration models that produce energetic nonthermal electrons in SNRs also accelerate ions, the total energy budget implied by these results would exceed the available energy of the SNR blast wave for $k_{e-p} \sim 10^{-2}$ if the remnant distance is $5 \mathrm{kpc}$.

For the smallest distance allowed by HI absorption measurements, an electron-to-proton ratio $k_{e-p}>0.7$ is required for the entire particle energy budget to not exceed $5 \times 10^{51} \mathrm{erg}$. Such high required values of $k_{e-p}$, along with the low cutoff energy and extremely sharp
TABLE 2

Model Parameters: $\gamma$-RAYS FROM SNR

\begin{tabular}{lcc}
\hline \hline Parameter & Hadronic Model & Leptonic Model \\
\hline$d$ (fixed) & $5 \mathrm{kpc}$ & $5 \mathrm{kpc}$ \\
$n_{0}$ & $6.8 \mathrm{~cm}^{-3}$ & $0.04 \mathrm{~cm}^{-3}$ \\
$\alpha_{e}\left(=\alpha_{p}\right)$ & 1.8 & 1.8 \\
$E_{c u t, e}$ & $0.9 \mathrm{TeV}$ \\
$E_{c u t, p}$ & $50 \mathrm{GeV}$ & $1 \mathrm{TeV}$ \\
$b^{\mathrm{a}}$ & $70 \mathrm{GeV}$ & 2.0 \\
$E_{e}^{\mathrm{b}}$ & 1.0 & $8 \times 10^{49} \mathrm{erg}$ \\
$E_{p}^{\mathrm{c}}$ & $5 \times 10^{48} \mathrm{erg}$ & $8 \times 10^{51} \mathrm{erg}$ \\
$k_{e-p}$ (fixed) & $5 \times 10^{50} \mathrm{erg}$ & $10^{-2}$ \\
$B$ & $10^{-2}$ & $2.0 \mu \mathrm{G}$ \\
& $10.0 \mu \mathrm{G}$ & \\
\hline
\end{tabular}

Note. -

a) Curvature index on exponential cut-off

b) Total electron energy

c) Total proton energy

spectral cutoff appear problematic. Thus, we conclude that a leptonic scenario in which the observed $\gamma$-ray emission from MSH 11-62 is dominated by IC emission is ruled out for all but the smallest possible distances, and still problematic at such small distance values.

\subsection{Gamma-rays from the $P W N$}

The X-ray and radio observations of MSH 11-62 clearly identify a powerful PWN in the system. The electrons responsible for the $\mathrm{X}$-ray and radio emission also produce $\gamma$-ray emission through inverse Compton scattering. To investigate the scenario in which the Fermi LAT emission is produced entirely by the PWN, we calculate the evolution of the composite system using the model of Gelfand, Slane, and Zhang (2009). We assume expansion of the SNR into a uniform medium of density $n_{0}$, driven by an ejecta mass $M_{e j}$. The PWN expansion is powered by the input of a central pulsar with a spin-down power that evolves as

$$
\dot{E}(t)=\dot{E}_{0}\left[1+\frac{t}{\tau_{0}}\right]^{-\frac{p+1}{p-1}}
$$

where $\tau_{0}$ is the initial spin-down timescale for the pulsar, and $p$ is the braking index. The PWN expansion is confined by the surrounding ejecta. Particles are injected into the nebula with a power law spectrum, and evolved through radiative and adiabatic losses. The emission spectrum is then calculated based upon the evolved magnetic field strength of the nebula at the age required to reproduce the observed radius of the SNR (Figure 9). The ambient radiation field was assumed to be a combination of the cosmic microwave background (CMB) and infrared photons from local dust.

The parameters of the model are summarized in Table 3 . We fixed the distance at $5 \mathrm{kpc}$ and assumed $E_{S N R}=10^{51} \mathrm{erg}$. We sampled values for $n_{0}$ in the range $0.02-0.08 \mathrm{~cm}^{-3}$ based on the estimates provided from the X-ray emission (see Section 3.4), and determined that the broadband spectrum is not highly sensitive to the ambient density, with flux values varying by only $\sim 30 \%$ in the X-ray band (see Figure 11). Braking index values over the range $p=2-3$, and ejecta mass values between $1.5-8.5 M_{\odot}$, produce flux variations similar in magnitude to those observed for the above range in $n_{0}$, and we 

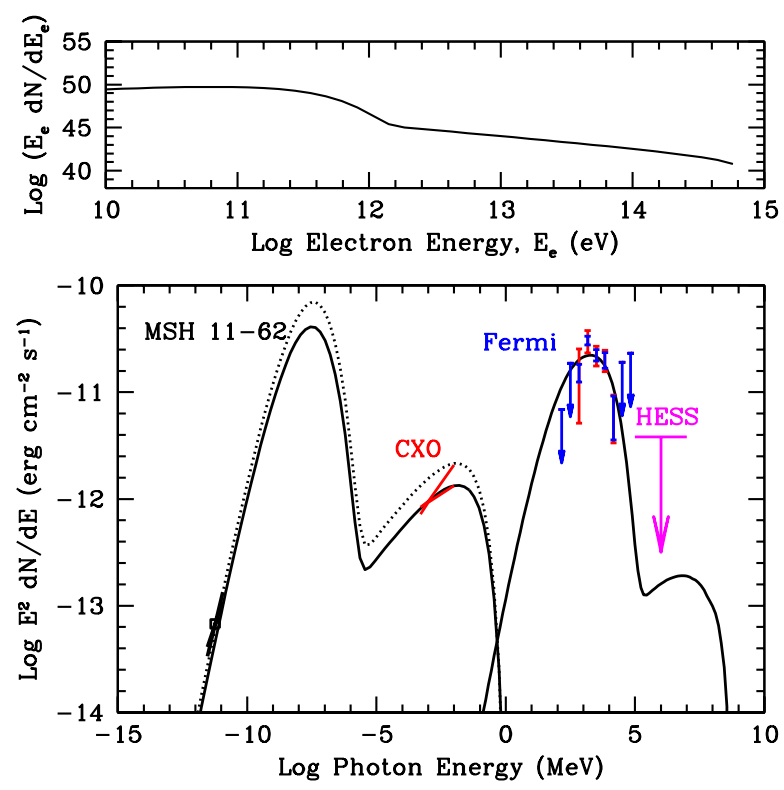

FIG. 11. - Models for the nonthermal emission from MSH 1162 (lower panel) under the assumption that the $\gamma$-ray emission is produced entirely by the PWN. Dashed, solid, and dotted lines correspond to $n_{0}=0.02,0.04$, and $0.08 \mathrm{~cm}^{-3}$. The upper panel shows the associated electron distribution, which is modeled as an evolved Maxwellian distribution with power law tail.

thus fixed these quantities at the values shown in Table 3. The observed radio flux provides an upper limit for the minimum electron energy; lower values of $E_{e, \text { min }}$ do not appreciably change the spectrum. Similarly, the X-ray spectrum provides a lower limit for the maximum electron energy. Higher values of $E_{e, \max }$ allow the photon spectrum to extend to higher energies, but the current data provide no additional constraints on this.

With the above parameters fixed, we varied values for the pulsar spin-down timescale, $\tau_{0}$, the initial spin-down energy, $\dot{E}_{0}$, the electron spectral index, $\alpha_{e}$, and the fraction of the pulsar wind energy in the form of magnetic fields and particles, $\eta_{B}$ and $\eta_{e}$ respectively. We found that it was not possible to reproduce the significant $\gamma$-ray emission in the Fermi LAT band with simple power law or broken power law models for the particle spectrum under these assumptions. We then investigated a model consisting of a Maxwellian electron distribution with a power law tail, corresponding to spectra observed in PIC simulations of relativistic shocks (Spitkovsky 2008), considering a range of values for the energy of the Maxwellian peak and for the fraction of the total electron energy found in the power law component. The temperature and energy density of the IR photon field were adjusted slightly to tune the agreement with the data. The final values listed along with the other model parameters in Table 3 are within reasonable expectations for this region of the Galaxy (see Strong et al. 2000).

As shown in Figure 11, we find that the broadband emission from MSH 11-62 can be reproduced to reasonable fidelity for the parameters shown in Table 3 . The upper panel of the Figure contains the electron spectrum, and the lower panel presents the synchrotron and IC emission along with the observed data. The radii for the SNR and PWN are shown in Figure 9, along with the PWN magnetic field. The age required to match
TABLE 3

Model Parameters: $\gamma$-RAYS From PWN or Pulsar

\begin{tabular}{lcc}
\hline \hline \multicolumn{1}{c}{ Parameter } & $\gamma$-rays from PWN & $\gamma$-rays from Pulsar \\
\hline Input: & & \\
$d$ (fixed) & $5 \mathrm{kpc}$ & $5 \mathrm{kpc}$ \\
$E_{S N R}$ (fixed) & $10^{51} \mathrm{erg}$ & $10^{51} \mathrm{erg}$ \\
$M_{e j}$ (fixed) & $2.5 M_{\odot}$ & $2.5 M_{\odot}$ \\
$n_{0}$ (fixed) & $0.04 \mathrm{~cm}^{-3}$ & $0.04 \mathrm{~cm}^{-3}$ \\
$p$ (fixed) & 3.0 & 3.0 \\
$\tau_{0}$ & $1000 \mathrm{yr}$ & $1000 \mathrm{yr}($ fixed) \\
$\dot{E}_{0}$ & $4 \times 10^{39} \mathrm{erg} \mathrm{s}^{-1}$ & $3 \times 10^{38} \mathrm{erg} \mathrm{s}^{-1}$ \\
$\eta_{e}^{\mathrm{a}}$ & 0.999 & 0.995 \\
$\eta_{B}^{\mathrm{b}}$ & 0.001 & 0.005 \\
$E_{e, \text { min }}$ & $0.1 \mathrm{GeV}$ & $1 \mathrm{MeV}$ \\
$E_{e, \text { max }}^{\mathrm{c}}$ & $900 \mathrm{TeV}$ & $8.5 \mathrm{PeV}$ \\
$E_{p}^{\mathrm{c}}$ & $100 \mathrm{GeV}$ & \\
$f_{P}^{\mathrm{d}} L$ & $.1 \%$ & $4.4 \mathrm{GeV}$ \\
$E_{b}^{\mathrm{e}}$ & & 0.0 \\
$\alpha_{e}$ & 2.33 & 2.7 \\
$\alpha_{e, 2}$ & & $25 \mathrm{~K}(\mathrm{fixed})$ \\
$T_{I R}^{\mathrm{f}}$ & $25 \mathrm{~K}$ & \\
$\left(W_{I R} / W_{C M B}\right)^{\mathrm{g}}$ & $2 W_{C M B}$ & \\
$D_{\text {Derived: }}$ & & $1300 \mathrm{yr}$ \\
Age & $1200 \mathrm{yr}$ & $7.3 \mu \mathrm{G}$ \\
$B_{P W N}$ & $6.1 \mu \mathrm{G}$ & \\
\hline
\end{tabular}

Note. -

a) Fraction of energy in electrons

b) Fraction of energy in magnetic field

c) Peak energy of Maxwellian electron component

d) Fraction of energy in power law component

e) Power law break energy

f) Effective temperature of IR photon field

g) Ratio of IR energy density relative to $\mathrm{CMB}$

the observed SNR radius is $\sim 1.2 \mathrm{kyr}$. The magnetic field of the PWN has declined to a value of $\sim 6 \mu \mathrm{G}$, and the PWN radius is approaching the stage at which it encounters the SNR reverse shock, as indicated by the subsequent decline in $R_{P W N}$. This appears qualitatively consistent with the X-ray and radio morphology, as discussed in Section 5.1, however the narrowest portion of the PWN is considerably smaller than indicated in Figure 9 . The very distorted shape of the nebula suggests that the reverse shock has propagated asymmetrically in MSH 11-62, which is not a scenario that can be treated in our spherical model.

The magnetic field in this scenario is far below the minimum-energy value calculated from radio measurements, indicating that the nebula is particle-dominated. We note that the $\sim 65 \mu \mathrm{G}$ field estimated by Harrus, Hughes, \& Slane (1998) was based on the assumption that the minimum-energy conditions hold in MSH 1162. Additional estimates based on the interpretation of the spectral break between the radio and X-ray bands as being an evolutionary effect, and on the pressure in the PWN being in equilibrium with that of the thermal gas in the SNR, led to even higher estimated values of the magnetic field. However, no modeling was performed to demonstrate which, if any, of those assumed conditions provide a self-consistent picture of the composite system. Here we have carried out such modeling efforts and find that a particle-dominated nebula is required, with the magnetic field at the current epoch being well below the equipartition value. Similar results are obtained for the evolved nebulae in W44 (Bucciantini et al. 2011), 
G0.9+0.1, and G338.3-0.0 (Fang \& Zhang 2010). We also note that even though different values for the assumed distance can lead to different values of the model parameters (see below), the observed X-ray spectral index constrains the magnetic field to be low, and the overall magnetization to be low as well.

According to this model, $\gamma$-ray emission in the Fermi LAT band is produced entirely by electrons in the Maxwellian peak of the spectrum. This is consistent with results for Vela X (de Jager, Slane, \& LaMassa 2008), HESS J1640-465 (Slane et al. 2010), and several other PWNe (Fang \& Zhang 2010) that suggest a low-energy electron component distinct from the power law component (although for Vela X this was modeled as an additional power law component). For MSH 11-62, we find a mean Lorentz factor of $\gamma=2 \times 10^{5}$ for the electrons in the Maxwellian component, with only $0.1 \%$ of the total energy in the power law tail.

We note that the initial spin-down power required for this model, $\dot{E}_{0}=4 \times 10^{39} \mathrm{erg} \mathrm{s}^{-1}$, is uncomfortably large. For the assumed braking index $p=3$, this would lead to a value of $\dot{E}=8.3 \times 10^{38} \mathrm{erg} \mathrm{s}^{-1}$ at the current epoch - higher than that for any known pulsar. This would imply $L_{x} / \dot{E} \sim 10^{-5}$ for the pulsar, which is extremely low.

Given that the distance to MSH 11-62 is not well known, the numerical values from the model above are quite uncertain. Changes in the assumed distance can be accommodated, at least in part, by adjusting the spindown power, for example. However, larger distance values lead to even higher values of $\dot{E}$. Reasonable fits to the broadband spectrum can also be obtained for a distance as low as $2 \mathrm{kpc}$, yielding a current spin-down power of $\sim 2 \times 10^{38} \mathrm{erg} \mathrm{s}^{-1}$. While still extremely high, this is similar to the value for PSR J2022+3842, a pulsar recently discovered in G76.9+1.0 (Arzoumanian et al. 2011). This pulsar is characterized by an extremely low conversion efficiency of spin-down power into X-rays as well, but the value $\left(L_{x} / \dot{E} \sim 6 \times 10^{-5}\right)$ is still much higher than that inferred for the pulsar in MSH 11-62 if the Fermi LAT emission is being produced by the PWN. We conclude that it is possible for the observed $\gamma$-ray emission from MSH 11-62 to be produced by the PWN, but that this would require a very significant amount of energy in the Maxwellian peak of the electron spectrum, leading to spin-down parameters for the pulsar that seem unlikely.

\subsection{Gamma-rays from the Pulsar}

Fermi observations have clearly established that pulsars comprise a significant fraction of the Galactic population of $\mathrm{GeV}$ point sources. The associated spectra are characterized by a power law with an exponential cutoff, with $E_{\text {cut }} \sim 1-6 \mathrm{GeV}$, and $\Gamma \sim 1-2$ (Abdo et al. 2010). This is very similar to the spectrum observed for MSH 11-62. Being associated with a composite SNR, for which our X-ray observations identify the underlying neutron star, it is quite possible that the observed $\gamma$-ray emission originates with the pulsar itself.

The ratio of the X-ray flux from the point source in MSH 11-62 to the observed $\gamma$-ray flux is $F_{x} / F_{\gamma} \sim 5 \times$ $10^{-3}$. This value is typical of that for pulsars detected by the Fermi LAT, although there is a large spread in

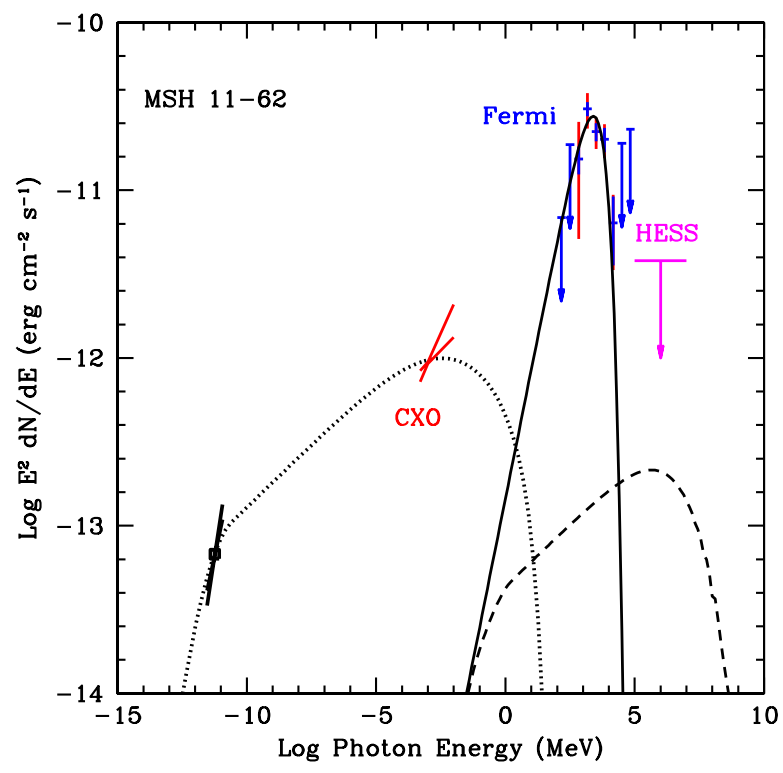

FIG. 12.- Model for the PWN emission from MSH 11-62 under the assumption that the $\gamma$-ray emission arises from the pulsar. Parameters for the model are given in Table 3.

this ratio for the population.

Using the approximate relationship $L_{x} \approx 10^{-3} \dot{E}$ derived from a variety of studies of X-ray $\mathrm{PWNe}$ and their associated pulsars, the spectrum of the PWN in MSH 11-62 suggests an underlying pulsar with $\dot{E} \sim$ $10^{37} d_{5}^{2} \mathrm{erg} \mathrm{s}^{-1}$. We note, however, that there are large variations in the observed relation between $L_{x}$ and $\dot{E}$ (e.g., Vink, Bamba, \& Yamazaki 2011). More than $85 \%$ of the pulsars detected by the Fermi LAT, and all of those pulsars discovered in the $\gamma$-ray band, have lower values of $\dot{E}$. However, of currently reported pulsars discovered in $\gamma$-rays, only one has a Fermi LAT flux lower than that associated with MSH 11-62. Thus, the lack of identified pulsations in any band is not strong evidence that the emission from MSH 11-62 is not associated with the pulsar itself. The marginal evidence for extension of the $\gamma$-ray emission, which would rule out the pulsar as the source of the emission, is of particular importance in this context, and requires revisiting in the future when additional data from this source have been obtained.

If we assume that the Fermi LAT emission arises entirely from the pulsar in MSH 11-62, the PWN radio and X-ray emission can then be reproduced by a broken power law electron spectrum with $\dot{E}_{0} \sim 3 \times 10^{38} \mathrm{erg} \mathrm{s}^{-1}$ and an age of $\sim 1300 \mathrm{yr}$, at which time the ambient density $n_{0}=0.04 \mathrm{~cm}^{-3}$ inferred from the SNR X-ray emission yields the observed radius for the SNR assuming a distance of $5 \mathrm{kpc}$. Here, as in Section 5.3, we have fixed the values for $d, M_{e j}, n_{0}$, and $p$. In addition, we have used the same model values for the IC photon fields as were used in Section 5.3, although these are largely irrelevant here since the IC emission falls well below the observed $\gamma$-ray emission. We have varied the remaining parameters in Table 3 in order to provide an approximate match to the observed radio and X-ray spectra. The resulting model spectrum is shown in Figure 12, and the parameter values for the model are listed in $\mathrm{Ta}$ ble 3. The PWN magnetic field strength is $\sim 7 \mu \mathrm{G}$, a 
factor of 5 lower than the minimum-energy field derived from radio observations, and the spin-down power at the current epoch is $\dot{E}=5.7 \times 10^{37} \mathrm{erg} \mathrm{s}^{-1}$. This leads to $L_{x} / \dot{E} \approx 2 \times 10^{-4}$, somewhat low, but well within the observed range for known pulsars. In this model, the SNR $\mathrm{RS}$ has not yet begun to impact the PWN, leaving the observed X-ray and radio morphology unexplained.

We note that this model again leads to a particledominated nebula, with the magnetic energy comprising only $\sim 4 \%$ of the total energy. Models that produce larger magnetic fields at the derived age can be obtained by increasing $\eta_{B}$. However this leads to a synchrotronloss cutoff below the X-ray band. Conversely, a better fit to the X-ray slope can be obtained by further reducing the fraction of wind energy that appears as magnetic flux. This, however, requires a much higher value of $\dot{E}_{0}$ and results in an even lower value for the current magnetic field strength in the PWN. In any event, the low magnetization of the nebula appears robust in these models, although we caution that our spherically-symmetric semianalytical calculations do not address more complex geometrical and magnetohydrodynamical effects that surely complicate the magnetic field structure in PWNe.

\section{CONCLUSIONS}

Radio observations of MSH 11-62 indicate that this is a composite SNR containing a central PWN. X-ray observations confirm this, with clear identifications of the PWN synchrotron emission as well as the presence of an X-ray point source that most likely corresponds to the pulsar driving the system. The X-ray spectral index in the PWN increases with distance from the compact source, as expected from synchrotron losses, and the strongly asymmetric morphology of the PWN suggests an interaction with the SNR RS.

Observations with the Fermi LAT reveal distinct $\gamma$ ray emission from MSH 11-62, but the limited angular resolution makes it impossible to ascertain the origin of the emission directly. We have considered three cases, with the $\gamma$-ray emission arising from the SNR, the PWN, and the pulsar, respectively. Models for each scenario can reproduce basic features of the observed broadband nonthermal emission, but not without difficulties. If the emission arises from the SNR, then a hadronic scenario requires an ambient density that is a factor of $\sim 100$ higher than that implied by the thermal X-ray emission, while a leptonic scenario requires that an unreasonably large fraction of the SNR mechanical energy is converted to relativistic particles unless the SNR distance is quite low. Both models require very low energy cutoffs in the particle spectra. If the emission arises from the PWN, then the input spectrum from the pulsar requires a dominant Maxwellian component, with only $0.1 \%$ of the energy in the accompanying power law tail, and an extremely high pulsar spin-down power that appears inconsistent with the observed X-ray and $\gamma$-ray flux values.

The most likely scenario is that the $\gamma$-ray emission arises from the pulsar itself. While models for the PWN emission still imply a somewhat high spin-down power, the lack of detected $\gamma$-ray pulsations is not surprising given the relatively low flux of the source. The weak evidence for extension in the $\gamma$-ray emission is not sufficient to reject this scenario, but is of considerable interest for additional study with future observations.

The authors thank the referee, Rino Bandiera, for his careful review and helpful commentary on this manuscript.

NASA supported this work via grant numbers NRA 00-OSS-07/03500279 and NGT5-159. PS acknowledges support from NASA Contract NAS8-03060. The ATCA is funded by the Commonwealth of Australia for operation as a National Facility managed by CSIRO.

RR, ML-G, and SF have participated as members of the Fermi LAT Collaboration, which acknowledges generous ongoing support from a number of agencies and institutes that have supported both the development and the operation of the LAT as well as scientific data analysis. These include the National Aeronautics and Space Administration and the Department of Energy in the United States, the Commissariat à l'Energie Atomique and the Centre National de la Recherche Scientifique / Institut National de Physique Nucléaire et de Physique des Particules in France, the Agenzia Spaziale Italiana and the Istituto Nazionale di Fisica Nucleare in Italy, the Ministry of Education, Culture, Sports, Science and Technology (MEXT), High Energy Accelerator Research Organization (KEK) and Japan Aerospace Exploration Agency (JAXA) in Japan, and the K. A. Wallenberg Foundation, the Swedish Research Council and the Swedish National Space Board in Sweden. Additional support for science analysis during the operations phase is gratefully acknowledged from the Istituto Nazionale di Astrofisica in Italy and the Centre National d'Études Spatiales in France.

\section{REFERENCES}

Abdo, A. A., et al. 2009, ApJ, 706, 1331

Abdo, A. A., et al. 2010, ApJS, 187, 460

Abdo, A. A. et al. 2011, arXiv:1108.1435 1

Arzoumanian, Z., et al. 2011, ApJ, 739, 39

Bucciantini, N., Arons, J., \& Amato, E. 2011, MNRAS, 410, 381

Bocchino, F., et al. 2001, A\&A, 369, 1078

Castro, D. \& Slane, P. 2010, ApJ, ApJ, 717, 372

de Jager, O., Slane, P., \& LaMassa, S. 2008, ApJ, 689, L125

Ellison, D. C., et al. 2010, ApJ, 712, 287

Fang, J. \& Zhang, L. 2010, A\&A, 515, A20

Gaensler, B. M. \& Slane, P. 2006, ARA\&A, 44, 17

Gelfand, J.D. et al. 2007, ApJ, 663, 468

Gelfand, J. D., Slane, P. O, \& Zhang, W. 2009, ApJ, 703, 2051

Grondin, M.-H., et al. 2011, ApJ, 738, 42

Harrus, I. M., Hughes, J. P., \& Slane, P. 1998, ApJ, 499, 273
Harrus, I. M., et al. 2002, Neutron Stars in Supernova Remnants, ASP Conference Series, Vol. 271, held in Boston, MA, USA, 14-17 August 2001. Edited by Patrick O. Slane and Bryan M. Gaensler. San Francisco: ASP, 2002., p.203

Hartman, R.C. et al. 1999, ApJS, 123, 79

Hoppe, S. 2008, Proceedings of the 30th International Cosmic Ray Conference Rogelio Caballero, Juan Carlos D'Olivo, Gustavo Medina-Tanco, Lukas Nellen, Federico A. Snchez, Jos F. Valds-Galicia (eds.) Universidad Nacional Autnoma de Mexico, Mexico City, Mexico, 2008 Vol. 2, pages 579-582

Johnston, S., McCure-Griffiths, N. M., \& Koribalski, B. 2004, MNRAS, 348, L19

Kargaltsev, O. \& Pavlov, G. 2008, AIP 983, 171

Kaspi, V. M., et al. 1997, ApJ, 485, 820

Kennel, C. F., \& Coroniti, F. V. 1984, ApJ, 283, 694 
Kerr, M. 2011, ApJ, 732, 38

Lazendic, J. S., et al. 2000, ApJ, 540, 808

Mattox, J. R., et al. 1996, ApJ, 461, 396

Moffett, D., Gaensler, B.M.; Green, A. 2001, Young Supernova

Remnants: Eleventh Astrophysics Conference. AIP Conference

Proceedings, Volume 565, pp. 333-336 (2001)

Moffett, D., Gaensler, B.M., Green, A., Slane, P., Harrus, I.M.,

Dodson, R. 2002, Neutron Stars in Supernova Remnants, ASP

Conference Series, Vol. 271, held in Boston, MA, USA, 14-17

August 2001. Edited by Patrick O. Slane and Bryan M.

Gaensler. San Francisco: ASP, 2002., p.221

Reynolds, S. P. 2003, Proceedings of IAU Colloquium 192, 10

Years of SN1993J (Valencia, Spain, April 2003) Edited by J.M.

Marcaide \& K.W. Weiler Springer-Verlag
Reynolds, S. P. 2008, ARA\&A, 46, 89

Roger, R. S., Milne, D. K., Caswell, J. L. and Little, A. G. 1986 MNRAS, 219, 815

Slane, P., et al. 2000, ApJ, 533, L295

Slane, P., et al. 2004, ApJ, 616, 403

Slane, P. et al. 2010, ApJ, 720, 266

Spitkovsky, A. 2008, ApJ, 682, L5

Strong, A. W., Moskalenko, I. V., \& Reimer, O. 2000, ApJ, 537, 763

Sturner, S. J. \& Dermer, C. D. 1995, A\&A, 293, L17

Tam, C., Roberts, M. S. E., \& Kaspi, V. M. 2002, ApJ, 572, 202

Vazquez, R. A. \& Feinstein, A. 1990, A\&AS, 86, 209

Vink, J., Bamba, A., \& Yamazaki, R., 2011, ApJ, 727, 131

Wilson, A. S. 1986, ApJ, 302, 718 